Article

\title{
Synthesis of 1-(para-methoxyphenyl)tetrazolyl-Substituted 1,2,3,4-Tetrahydroisoquinolines and Their Transformations Involving Activated Alkynes
}

\author{
Alexander A. Titov ${ }^{1}$, Reza Samavati ${ }^{1}$, Elena V. Alexandrova ${ }^{1}$, Tatiana N. Borisova ${ }^{1}$, \\ Tuyet Anh Dang Thi ${ }^{2}$, Van Tuyen Nguyen ${ }^{2,3}$, Tuan Anh Le ${ }^{4} \mathbb{0}$, Alexey V. Varlamov ${ }^{1}$, \\ Erik V. Van der Eycken ${ }^{1,5}$ and Leonid G. Voskressensky ${ }^{1, *}$ \\ 1 Organic Chemistry Department, Peoples' Friendship University of Russia (RUDN University), \\ 6 Miklukho-Maklaya St., 117198 Moscow, Russia; titov_aa@pfur.ru (A.A.T.); reza_nasa3@yahoo.com (R.S.); \\ elena-aleksandrova-00-lena@mail.ru (E.V.A.); borisova_tn@pfur.ru (T.N.B.); \\ avarlamov@sci.pfu.edu.ru (A.V.V.); erik.vandereycken@kuleuven.be (E.V.V.d.E.) \\ 2 Institute of Chemistry of Vietnam Academy of Science and Technology, 18 Hoang Quoc Viet, Cau Giay, \\ Hanoi 100000, Vietnam; dangtuyetanh1201@gmail.com (T.A.D.T.); ngvtuyen@hotmail.com (V.T.N.) \\ 3 Graduate University of Science and Technology, Vietnam Academy of Science and Technology, \\ 18 Hoang Quoc Viet, Cau Giay, Hanoi 100000, Vietnam \\ 4 Faculty of Chemistry of VNU University of Science, 19 Le Thanh Tong, Hoan Kiem, Hanoi 100000, Vietnam; \\ huschemical.lab@gmail.com \\ 5 Laboratory for Organic \& Microwave-Assisted Chemistry (LOMAC), Department of Chemistry, \\ KU Leuven Celestijnenlaan 200F, 3001 Leuven, Belgium \\ * Correspondence: lvoskressensky@sci.pfu.edu.ru; Tel.: +7-495-955-0729
}

Academic Editor: Wei Zhang

Received: 10 October 2018; Accepted: 15 November 2018; Published: 17 November 2018

\begin{abstract}
Methoxyphenyl)tetrazolyl-substituted 6,7-dimethoxy(6,7-methylenedioxy)-1,2,3,4tetrahydroisoquinolines formed tetrazolyl-substituted azocines in high yields by using activated alkynes. Unsubstituted at 6,7,8-aromatic fragment 1-tetrazolylisoquinoline interacted in several pathways forming tetrazolyl-substituted azocines, 1-tetrazolyl-1-R-vinylisoquinolines and 3-azaspiro[5.5]undeca-1,7,9-triene.
\end{abstract}

Keywords: tetrazolylisoquinolines; tetrazolyl benzazocines; cycle expansion; Stevens rearrangement; activated alkynes

\section{Introduction}

Multicomponent processes are an accessible tool for creating molecular diversity, have a high synthetic potential, and show high atom and step economy. There are many examples of isonitriles in multicomponent reactions for forming original biologically-active compounds [1,2]. They are widely used in green chemistry [3-5] and in the synthesis of tetrazoles [6-11]. Substituted or heterocyclic annulated tetrazoles have an extensive range of biological activity. They have exhibited anti-inflammatory [12], analgesic [12], and anticancer [13] activities. Substituted benzazocines and azocinoindoles can inhibit acetyl- and butyrylcholinesterases [14,15], and they are promising compounds for creating antineurodegenerative drugs. The combination of two pharmacophore fragments of azocine and tetrazole in one molecule can enhance previously identified, or create new activities. 


\section{Results}

Recently, we reported examples of cotarnine chloride and 2-methyl-3,4-dihydro- $\beta$-carbolinium iodide that iminium salts, can be used in multicomponent Ugi reactions [16,17]. At the 1-position of the methyl, phenyl, or benzyl substituent in the iminium salts of isoquinolines, the Ugi azido reaction is not realized. Herein, we propose to determine whether substituents in the aromatic fragment of 1-tetrazolyl-substituted isoquinolines affect the direction of transformations involving activated alkynes. Specifically, we propose the synthesis of 1-( $p$-methoxyphenyltetrazolyl)-1,2,3,4-tetrahydroisoquinoline, and its 6,7-dimethoxy- and 6,7-(methylenedioxy)-8-methoxy derivatives 1a-c [16].

We prepared tetrazolyl-substituted isoquinolines, by reacting the corresponding iminium salt with $p$-methoxyphenyl isonitrile and sodium azide (Scheme 1).
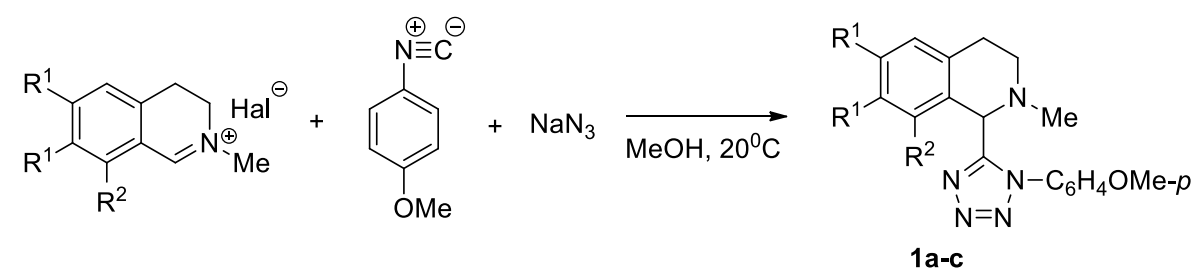

$$
\begin{aligned}
& \text { 1a } R^{1}=O M e, R^{2}=H(62 \%) \\
& \text { 1b } R^{1}=O C H_{2} O, R^{2}=O M e(94 \%) \\
& \text { 1c } R^{1}=R^{2}=H(65 \%)
\end{aligned}
$$

Scheme 1. Synthesis of tetrazolyl-substituted isoquinolines 1a-c via the Ugi azido reaction.

The tetrazolyl-substituted isoquinolines $\mathbf{1 a}, \mathbf{1 b}$, with oxygene-containing substituents in the aromatic moiety, reacted with methyl propiolate, acetylacetylene, and DMAD (dimethyl acetylenedicarboxylate) in trifluoroethanol at $20{ }^{\circ} \mathrm{C}$ for 1-12 days, affording tetrazolyl-substituted azocines $\mathbf{2 a - f}$ in nearly quantitative yields (Scheme 2 ).

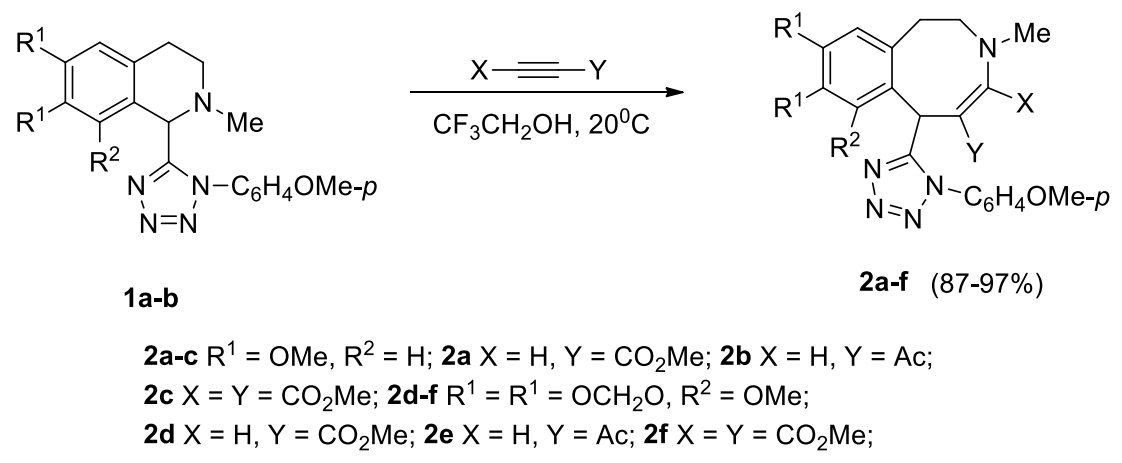

Scheme 2. Synthesis of tetrazolyl-substituted benzazocines $\mathbf{2 a}-\mathbf{f}$.

Previously, we obtained 6-(1-benzyl and 1-(2-ethyl-6-methylphenyl) tetrazolyl-substituted azocines from 1-tetrazolyl-2-methyl-6,7-methylenedioxy-8-methoxy-1,2,3,4-tetrahydroisoquinoline with activated alkynes, in yields of $64-75 \%$, and with DMAD, in a yield of $94 \%$ [16].

Unsubstituted at the aromatic fragment, tetrahydroisoquinoline 1c reacted difficultly and ambiguously with activated alkynes. The reaction with DMAD occurred at $20{ }^{\circ} \mathrm{C}$ over the course of 2 days (Scheme 3). In contrast, the reaction with methyl propiolate and acetylacetylene required heating to the boiling point of the solvent (Scheme 4). 


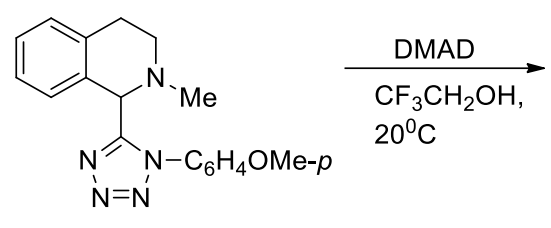

$1 \mathrm{c}$

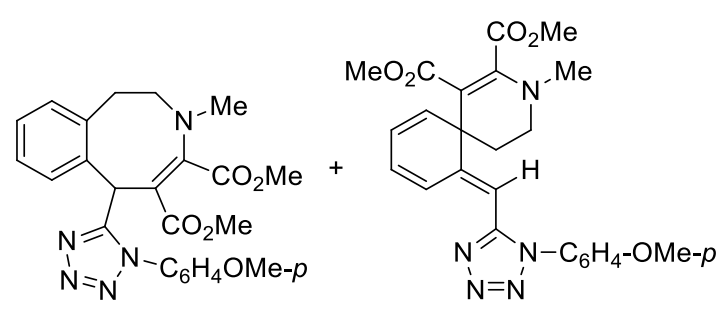

$2 \mathrm{~g}(50 \%)$

$3(17 \%)$

Scheme 3. Interaction of isoquinoline 1c with DMAD.

Schemes 3 and 4 show transformations of isoquinoline $1 \mathrm{c}$ with activated alkynes. Tetrazolyl-substituted benzazocines $\mathbf{2} \mathbf{g}$, $\mathbf{2 h}$ formed in the reactions of compound $\mathbf{1 c}$ with methyl propiolate and DMAD. We synthesized the Stevens rearrangement's products $4 \mathbf{a}, \mathbf{4 b}$, using methyl propiolate and acetylacetylene, via ylides as intermediates. It was unusual to obtain spiro compound 3 from the interaction with DMAD. Previously, we reported that such spiro compounds form in the reaction of activated alkynes with tetrahydropyridines $[c]$-connected with a five-membered heterocycle, with one heteroatom condensed with a benzo- or hetaryl-containing fragment $[18,19]$.

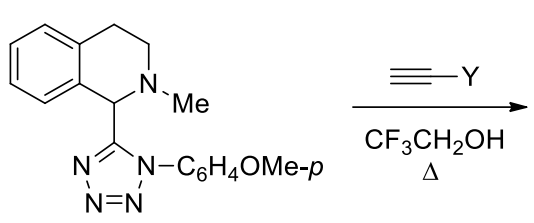

$1 \mathrm{c}$

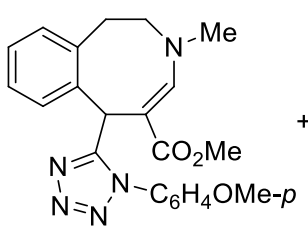

2h $(22 \%)$

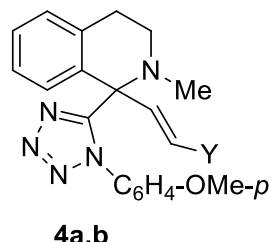

$4 a, b$

4a $\mathrm{Y}=\mathrm{CO}_{2} \mathrm{Me}(10 \%)$,

4b $Y=A c(17 \%)$.

Scheme 4. Interaction of isoquinoline 1c with activated terminal alkynes.

We assume that the conversion of tetrazolyl-substituted isoquinolines proceeds through zwitterion A, which is in equilibrium with the open form B (Scheme 5). An interaction of ions in the intermediate $\mathbf{B}$ leads to the formation of compounds $\mathbf{2} \mathbf{a}-\mathbf{h}$. The same products can be obtained as a result of nucleophilic attack of the cationic center in zwitterion $\mathbf{A}$ on position 1 of isoquinoline. In the reactions of methyl propiolate and acetylacetylene, the anionic center in ion $\mathbf{A}$ has a greater basicity than in the reaction of DMAD, and it can remove a proton from the C-1 position, forming ylide $\mathbf{C}$, the rearrangement of which leads to the synthesis of vinyl-substituted tetrazolylisoquinolines $\mathbf{4 a}, \mathbf{4 b}$.

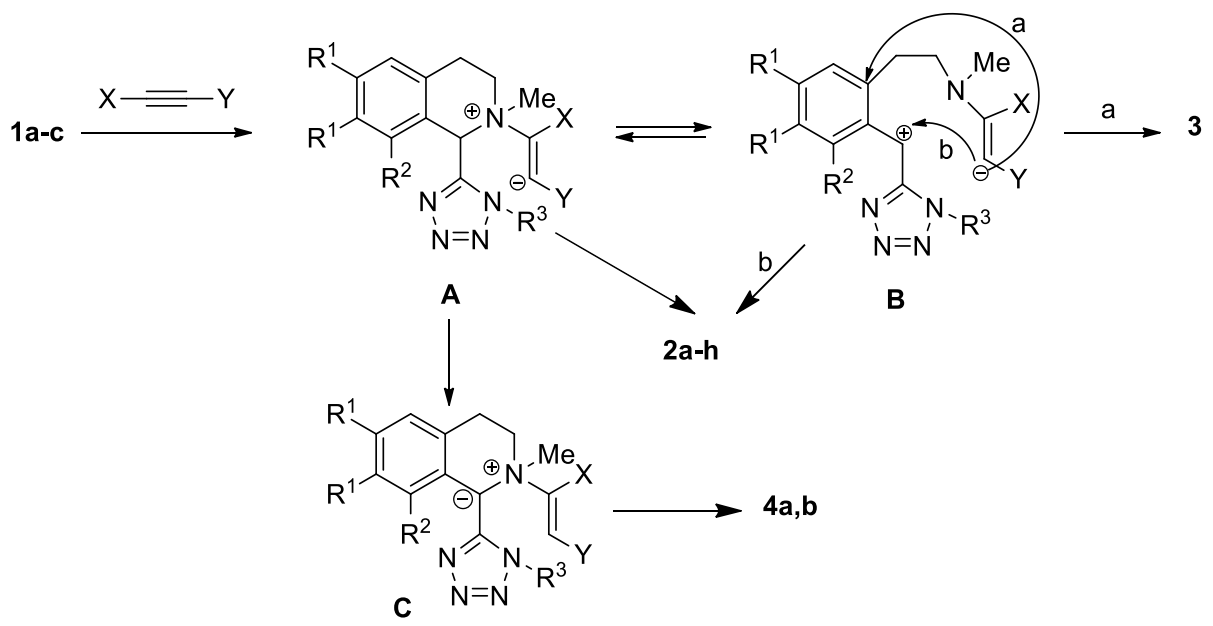

Scheme 5. Proposed mechanism for the formation of the isolated products 2-4. 
The formation of the unusual transformation product with DMAD (spiro compound 3) is probably associated with a more efficient delocalization of the anionic center in intermediate $\mathbf{B}$, which enables ipso-attack on the aromatic fragment.

We confirmed the structures of all synthesized compounds by spectral data. We characterized the ${ }^{1} \mathrm{H}-\mathrm{NMR}$ spectra of all tetrazolyl-substituted azocines by the presence of the weakfield singlets of H-6 protons at $\delta$ 6.30-6.01 ppm, and the spectra of azocines $\mathbf{2 a}, \mathbf{2} \mathbf{b}, \mathbf{2} \mathbf{d}, \mathbf{2} \mathbf{e}, \mathbf{2} \mathbf{g}$ by the presence of singlets at $\delta 7.45-7.26 \mathrm{ppm}$, due to the presence of enamine proton $\mathrm{H}-4$. The ${ }^{1} \mathrm{H}-\mathrm{NMR}$ spectra of isoquinolines $\mathbf{4 a}, \mathbf{4 b}$ exhibits two doublets, $\delta 7.83-7.73 \mathrm{ppm}$ and $\delta 5.54-5.35 \mathrm{ppm}$, with $J=16.2 \mathrm{~Hz}$, indicating transposition of protons at a double bond (See the Supplementary Materials for further details). We confirmed the structure of spiro-compound 3 by X-ray analysis (Figure 1).

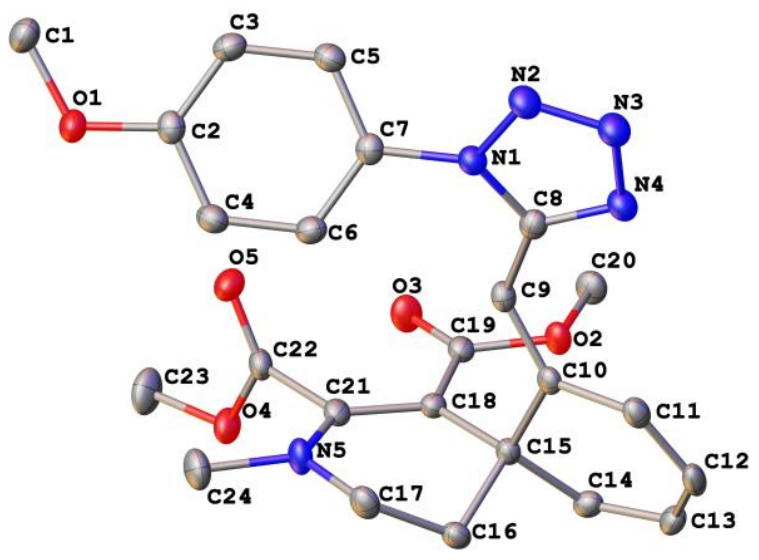

Figure 1. X-ray crystal diffraction of compound 3.

The tetrahydropyridine ring of the spiro compound 3 assumes a slightly distorted "sofa" conformation, with the carbon atom $\mathrm{C}(16)$ extending from the plane formed by the remaining atoms of the ring by $0.658 \AA$. Carbonyl fragment $\mathrm{C}(19)-\mathrm{O}(3)$ of the ester group is essentially coplanar with the basal plane of the tetrahydropyridine ring $C(15)-C(18)=C(21)-N(5)-C(17)$ (the dihedral angle is equal to $6.40^{\circ}$ ), due to bond conjugation. The nitrogen atom $\mathrm{N}(5)$ has a trigonal planar configuration (the sum of the valence angles is $359.7^{\circ}$ ). The cyclohexadiene ring of the spiro compound 3 assumes a slightly distorted "sofa" conformation, with the carbon atom $\mathrm{C}(15)$ extending from the formed by the remaining atoms of the ring by $0.444 \AA$. The 4-methoxyphenyl substituent in molecule 3 is twisted with a tetrazole ring at an angle of $34.20^{\circ}$. The molecule of spiro compound 3 contains the asymmetric carbon atom $\mathrm{C}(15)$. The molecules of compound 3 in the crystal form centrosymmetric dimers, due to two intermolecular hydrogen bonds $\mathrm{C}(5)-\mathrm{H}(5) \cdots \mathrm{O}(5)^{*}$.

To conclude, oxygen-containing substituents in the aromatic fragment at the tetrazolyl-substituted tetrahydroisoquinolines promote the formation of tetrazolylbenzazocines. Unsubstituted isoquinoline interacts in a more complex manner, and via several pathways.

\section{Experimental Section}

IR spectra were registered on a Fourier spectrometer Infralum FT-801 in KBr pellets (ISP SB RAS, Novosibirsk, Russia). ${ }^{1} \mathrm{H}$ and ${ }^{13} \mathrm{C}-\mathrm{NMR}$ spectra were acquired on a JEOL JNM-ECA 600 spectrometer (JEOL Ltd., Tokyo, Japan) (with operating frequencies of 600 and $150 \mathrm{MHz}$, respectively) in $\mathrm{CDCl}_{3}$ and DMSO- $d_{6}$ solution at $23^{\circ} \mathrm{C}$. Signal of the residual protons of the solvent $\left(7.26 \mathrm{ppm}\right.$ for $\left.\mathrm{CHCl}_{3}\right)$ was used as the reference in ${ }^{1} \mathrm{H}-\mathrm{NMR}$ spectra, while solvents signals (77.2 ppm for $\mathrm{CDCl}_{3}, 39.4$ ppm for DMSO- $d_{6}$ ) were used as the reference in ${ }^{13} \mathrm{C}-\mathrm{NMR}$ spectra. Mass spectra were recorded with LCMS-8040 Triple quadrupole liquid chromatograph mass-spectrometer from Shimadzu (Shimadzu Corporation, Tokyo, Japan). Elemental analysis was performed on a EuroVector EA-3000 elemental Analyzer (Eurovector, S.p.A., Milan, Italy). Melting points were determined by the open capillary method on a Stuart SMP10 
apparatus (Bibby Sterilin Ltd., Stone, UK). X-ray diffraction data were obtained on an automatic three-circle Bruker APEX-II CCD diffractometer (Bruker AXS GmbH, Karlsruhe, Germany). Sorbfil PTX-AF-A-UV plates (Imid Ltd., Krasnodar, Russia) were used for thin-layer chromatography and visualization with the iodine vapor. Column chromatography was performed on silica gel 40-60 $\mu \mathrm{m}$, 60 Å. DMAD, methyl propiolate, acetylacetylene (Acros Organics, Geel, Belgium) and trifluoroethanol (SIA “P\&M-Invest" Ltd., Moscow, Russia) were used without further purification. All solvents were distilled before use.

\subsection{Synthesis of 1-Tetrazolyl-Substituted Isoquinolines 1a-c (General Method)}

Sodium azide $(0.72 \mathrm{~g}, 11 \mathrm{mmol})$ was added to a solution of cotarnine chloride (2.12 $\mathrm{g}$, $8.3 \mathrm{mmol}$ ) or 6,7-dimethoxy-2-methyl-3,4-dihydroisoquinolinium iodide $(2.77 \mathrm{~g}$, $8.3 \mathrm{mmol})$ or 2-methyl-3,4-dihydroisoquinolinium iodide $(2.27 \mathrm{~g}, 8.3 \mathrm{mmol})$ and $p$-methoxyphenyl isonitrile (1.49 $\mathrm{g}$, $11 \mathrm{mmol})$ in methanol $(25 \mathrm{~mL})$ at $20^{\circ} \mathrm{C}$. Stirring was continued at room temperature, monitoring of the reaction progress was performed by TLC (EtOAc-hexane, 1:2). Cotarnine chloride and dimethoxy isoquinolinium iodide react for 1 day; unsubstituted aromatic fragment isoquinolinium iodide reacts for 2 days. The methanol was removed in vacuo, the residue was crystallized from a mixture of EtOAc-hexane (1:2) to afford 1-tetrazolyl-substituted isoquinolines 1a-c.

6,7-Dimethoxy-1-[1-(4-methoxyphenyl)-1H-tetrazol-5-yl]-2-methyl-1,2,3,4-tetrahydroisoquinoline (1a), Yield $1.96 \mathrm{~g}$ (62\%); beige solid; m.p. $=108-110{ }^{\circ} \mathrm{C} ; R_{\mathrm{f}}=0.48($ EtOAc-hexane, $1: 1) ;{ }^{1} \mathrm{H}-\mathrm{NMR}(600 \mathrm{MHz}$, $\left.\mathrm{CDCl}_{3}\right): \delta 6.95(\mathrm{~d}, J=7.8 \mathrm{~Hz}, 2 \mathrm{H}, \mathrm{H}-\mathrm{Ar}), 6.75(\mathrm{~d}, J=7.8 \mathrm{~Hz}, 2 \mathrm{H}, \mathrm{H}-\mathrm{Ar}), 6.42$ (s, 1H, H-8), 6.15 (s, $1 \mathrm{H}$, H-5), 5.05 (s, 1H, H-1), 3.79 (s, 6H, $\left.\mathrm{OCH}_{3}\right), 3.64$ (s, 3H, $\left.\mathrm{OCH}_{3}\right), 2.94-2.91\left(\mathrm{~m}, 1 \mathrm{H}, 3-\mathrm{CH}_{2}\right), 2.53-2.49$ $\left(\mathrm{m}, 1 \mathrm{H}, 3-\mathrm{CH}_{2}\right), 2.46-2.43\left(\mathrm{~m}, 2 \mathrm{H}, 4-\mathrm{CH}_{2}\right), 2.25\left(\mathrm{~s}, 3 \mathrm{H}, \mathrm{N}-\mathrm{CH}_{3}\right) ;{ }^{13} \mathrm{C}-\mathrm{NMR}\left(150 \mathrm{MHz}, \mathrm{CDCl}_{3}\right): \delta 160.3$, 156.6, 148.2, 147.7, 127.3, 127.1 (3C), 123.9, 113.5 (2C), 110.9, 108.9, 60.1, 55.9, 55.8, 55.5, 51.1, 43.7, 28.1; LCMS m/z: $382[\mathrm{M}+\mathrm{H}]^{+}$. Elemental analysis: calcd. for $\mathrm{C}_{20} \mathrm{H}_{23} \mathrm{~N}_{5} \mathrm{O}_{3} \mathrm{C} 62.98, \mathrm{H} 6.08, \mathrm{~N} 18.36 \%$, found C $62.72, \mathrm{H} 5.85, \mathrm{~N} 18.47 \%$.

4-Methoxy-5-[1-(4-methoxyphenyl)-1H-tetrazol-5-yl]-6-methyl-5,6,7,8-tetrahydro[1,3]dioxolo[4,5-g]isoquinoline (1b), Yield $3.09 \mathrm{~g}$ (94\%); beige solid; m.p. = 143-145 ${ }^{\circ} \mathrm{C} ; R_{\mathrm{f}}=0.41$ (EtOAc-hexane, 2:1); ${ }^{1} \mathrm{H}-\mathrm{NMR}$ $\left(600 \mathrm{MHz}, \mathrm{CDCl}_{3}\right): \delta 7.55-7.52(\mathrm{~m}, 2 \mathrm{H}, \mathrm{H}-\mathrm{Ar}), 7.06-7.03(\mathrm{~m}, 2 \mathrm{H}, \mathrm{H}-\mathrm{Ar}), 6.34(\mathrm{~s}, 1 \mathrm{H}, \mathrm{H}-9)$, $5.85\left(\mathrm{~d}, J=1.4 \mathrm{~Hz}, 1 \mathrm{H}, 2-\mathrm{CH}_{2}\right), 5.81\left(\mathrm{~d}, J=1.4 \mathrm{~Hz}, 1 \mathrm{H}, 2-\mathrm{CH}_{2}\right), 5.04(\mathrm{~s}, 1 \mathrm{H}, \mathrm{H}-5), 3.89\left(\mathrm{~s}, 3 \mathrm{H}, \mathrm{OCH}_{3}\right)$, $3.62\left(\mathrm{~s}, 3 \mathrm{H}, \mathrm{OCH}_{3}\right), 3.24-3.19\left(\mathrm{~m}, 1 \mathrm{H}, 7-\mathrm{CH}_{2}\right), 2.90-2.85\left(\mathrm{~m}, 1 \mathrm{H}, 8-\mathrm{CH}_{2}\right), 2.68-2.65\left(\mathrm{~m}, 1 \mathrm{H}, 7-\mathrm{CH}_{2}\right)$, 2.63-2.59 (m, 1H, 8-CH $), 2.22(\mathrm{~s}, 3 \mathrm{H}, \mathrm{N}-\mathrm{CH} 3) ;{ }^{13} \mathrm{C}-\mathrm{NMR}\left(150 \mathrm{MHz}, \mathrm{CDCl}_{3}\right): \delta 160.9,155.9,149.0,139.9$, 134.1, 129.3, 127.4, 127.1 (2C), 117.2, 114.7 (2C), 103.2, 100.9, 58.9, 55.8, 52.7, 46.3, 42.1, 25.6; LCMS m/z: $396[\mathrm{M}+\mathrm{H}]^{+}$. Elemental analysis: calcd. for $\mathrm{C}_{20} \mathrm{H}_{21} \mathrm{~N}_{5} \mathrm{O}_{4} \mathrm{C} 60.75, \mathrm{H} 5.35, \mathrm{~N} 17.71 \%$, found C 60.51, H 5.01, N $17.60 \%$.

1-[1-(4-Methoxyphenyl)-1H-tetrazol-5-yl]-2-methyl-1,2,3,4-tetrahydroisoquinoline (1c), Yield $1.84 \mathrm{~g}(69 \%)$; yellow solid; m.p. $=90-92{ }^{\circ} \mathrm{C} ; R_{\mathrm{f}}=0.59$ (EtOAc-hexane, 1:2); ${ }^{1} \mathrm{H}-\mathrm{NMR}\left(600 \mathrm{MHz}, \mathrm{CDCl}_{3}\right): \delta 7.09$ $(\mathrm{t}, J=7.7 \mathrm{~Hz}, 1 \mathrm{H}, \mathrm{H}-\mathrm{Ar}), 7.03(\mathrm{t}, J=7.4 \mathrm{~Hz}, 1 \mathrm{H}, \mathrm{H}-\mathrm{Ar}), 6.96(\mathrm{~d}, J=7.4 \mathrm{~Hz}, 1 \mathrm{H}, \mathrm{H}-\mathrm{Ar}), 6.93-6.91(\mathrm{~m}, 2 \mathrm{H}$, H-Ar), 6.77-6.75 (m, 2H, H-Ar), 6.72 (d, J = 7.7 Hz, 1H, H-Ar), 5.15 (s, 1H, H-1), 3.81 (s, 3H, OCH $)_{\text {), }}$ 3.00-2.95 (m, 1H, 3-CH $)$, 2.59-2.53 (m, 3H, 3,4-CH $), 2.29$ (s, 3H, N-CH3); ${ }^{13} \mathrm{C}-\mathrm{NMR}\left(150 \mathrm{MHz}, \mathrm{CDCl}_{3}\right)$ : $\delta$ 160.4, 156.6, 134.8, 132.4, 128.6, 127.3 (2C), 127.1, 126.6 (2C), 126.2, 113.5 (2C), 60.3, 55.5, 51.1, 43.7, 28.5; LCMS m/z: $322[\mathrm{M}+\mathrm{H}]^{+}$. Elemental analysis: calcd. for $\mathrm{C}_{18} \mathrm{H}_{19} \mathrm{~N}_{5} \mathrm{O} \mathrm{C}$ 67.27, $\mathrm{H}$ 5.96, $\mathrm{N} 21.79 \%$, found C 66.95, H 6.20, N 21.91\%.

\subsection{The Interaction of Isoquinolines 1a, $\mathbf{1 b}$ with Activated Alkynes (General Method)}

Alkynes (DMAD, methyl propiolate or acetylacetylene) $(4 \mathrm{mmol})$ was added to a solution of isoquinolines $\mathbf{1 a}$ or $\mathbf{1 b}(2 \mathrm{mmol})$ in trifluoroethanol $(10 \mathrm{~mL})$. The reaction mixture was kept at $20{ }^{\circ} \mathrm{C}$, isoquinolines $\mathbf{1 a}, \mathbf{1 b}$ reacted with methyl propiolate for 1 day, with acetylacetylene for $5 \mathrm{~h}$ and 10 days, respectively, with DMAD for 5 and 12 days, respectively. The reaction progress was monitored by 
TLC (sorbphil, EtOAc-hexane, 1:2). The solvent was evaporated in vacuum and the residue was recrystallized from EtOAc-hexane mixture.

Methyl (4E)-8,9-dimethoxy-6-[1-(4-methoxyphenyl)-1H-tetrazol-5-yl]-3-methyl-1,2,3,6-tetrahydro-3-benzazocin5-carboxylate (2a), Yield $0.90 \mathrm{~g}$ (97\%); white solid; m.p. $=152-154{ }^{\circ} \mathrm{C} ; R_{\mathrm{f}}=0.41$ (EtOAc-hexane, 2:1); IR (KBr) v $1635 \mathrm{~cm}^{-1}(\mathrm{C}=\mathrm{O}) ;{ }^{1} \mathrm{H}-\mathrm{NMR}\left(600 \mathrm{MHz}, \mathrm{CDCl}_{3}\right): \delta 7.43(\mathrm{~s}, 1 \mathrm{H}, \mathrm{H}-4), 7.08(\mathrm{~d}, J=8.7 \mathrm{~Hz}, 2 \mathrm{H}$, H-Ar), 6.85 (d, J = 8.7 Hz, 2H, H-Ar), 6.57 (s, 1H, H-7), 6.32 (s, 1H, H-10), 6.00 (s, 1H, H-6), 3.98-3.93 $\left(\mathrm{m}, 1 \mathrm{H}, 2-\mathrm{CH}_{2}\right), 3.81\left(\mathrm{~s}, 6 \mathrm{H}, \mathrm{OCH}_{3}\right), 3.66\left(\mathrm{~s}, 3 \mathrm{H}, \mathrm{OCH}_{3}\right), 3.54\left(\mathrm{~s}, 3 \mathrm{H}, \mathrm{OCH}_{3}\right), 3.06-2.96\left(\mathrm{~m}, 3 \mathrm{H}, 1,2-\mathrm{CH}_{2}\right)$, 2.93 (s, 3H, N-CH3); ${ }^{13} \mathrm{C}-\mathrm{NMR}\left(150 \mathrm{MHz}, \mathrm{CDCl}_{3}\right): \delta 169.4,160.8,159.6,153.6,147.5,147.3,128.7,127.9$, 127.7 (2C), 126.6, 115.3, 114.6, 114.1 (2C), 94.0, 55.8, 55.6, 55.5, 51.4, 51.2, 44.2, 40.8, 35.1; LCMS m/z: 466 $[\mathrm{M}+\mathrm{H}]^{+}$. Elemental analysis: calcd. for $\mathrm{C}_{24} \mathrm{H}_{27} \mathrm{~N}_{5} \mathrm{O}_{5} \mathrm{C} 61.92, \mathrm{H} 5.85, \mathrm{~N} 15.04 \%$, found C 61.70, $\mathrm{H} 6.01$, $\mathrm{N} 14.90 \%$.

1-\{(4E)-8,9-Dimethoxy-6-[1-(4-methoxyphenyl)-1H-tetrazol-5-yl]-3-methyl-1,2,3,6-tetrahydro-3-benzazocine-5yllethanone (2b), Yield $0.78 \mathrm{~g}(87 \%)$; beige solid; m.p. $=194-195^{\circ} \mathrm{C} ; R_{\mathrm{f}}=0.26$ (EtOAc- hexane, 2:1); IR $(\mathrm{KBr}) \vee 1620 \mathrm{~cm}^{-1}(\mathrm{C}=\mathrm{O}) ;{ }^{1} \mathrm{H}-\mathrm{NMR}\left(600 \mathrm{MHz}, \mathrm{CDCl}_{3}\right): \delta 7.32(\mathrm{~s}, 1 \mathrm{H}, \mathrm{H}-4), 7.10(\mathrm{~d}, J=9.0 \mathrm{~Hz}, 2 \mathrm{H}$, $\mathrm{H}-\mathrm{Ar}), 6.84(\mathrm{~d}, J=9.0 \mathrm{~Hz}, 2 \mathrm{H}, \mathrm{H}-\mathrm{Ar}),, 6.53(\mathrm{~s}, 1 \mathrm{H}, \mathrm{H}-7), 6.40$ (s, 1H, H-10), 6.25 (s, 1H, H-6), 4.24-4.19 $\left(\mathrm{m}, 1 \mathrm{H}, 2-\mathrm{CH}_{2}\right), 3.81\left(\mathrm{~s}, 6 \mathrm{H}, \mathrm{OCH}_{3}\right), 3.63\left(\mathrm{~s}, 3 \mathrm{H}, \mathrm{OCH}_{3}\right), 3.10\left(\mathrm{ddd}, J=15.5,6.6,2.7 \mathrm{~Hz}, 1 \mathrm{H}, 2-\mathrm{CH}_{2}\right), 3.01$ (s, 3H, N-CH3), 2.98-2.93 (m, 2H, 1-CH 2 ), 2.07 (s, 3H, $\left.\mathrm{COCH}_{3}\right) ;{ }^{13} \mathrm{C}-\mathrm{NMR}\left(150 \mathrm{MHz}, \mathrm{CDCl}_{3}\right): \delta 193.3$; 160.7, 159.5, 155.8, 147.4, 147.3, 128.4, 128.0, 127.6 (2C), 126.6, 115.5, 114.5, 114.0 (2C), 108.5, 55.8, 55.54, 55.51, 51.2, 44.4, 38.0, 34.7, 24.7; LCMS $m / z: 450[\mathrm{M}+\mathrm{H}]^{+}$. Elemental analysis: calcd. for $\mathrm{C}_{24} \mathrm{H}_{27} \mathrm{~N}_{5} \mathrm{O}_{4}$ C 64.13, H 6.05, N 15.58\%, found C 63.92, H 6.25, N $15.41 \%$.

Dimethyl (4E)-8,9-dimethoxy-6-[1-(4-methoxyphenyl)-1H-tetrazol-5-yl]-3-methyl-1,2,3,6-tetrahydro-3-benzazocin4,5-dicarboxylate (2c), Yield $0.97 \mathrm{~g}(93 \%)$; beige solid; m.p. $=108-110^{\circ} \mathrm{C} ; R_{\mathrm{f}}=0.47$ (EtOAc-hexane, 1:1); IR $(\mathrm{KBr}) \vee$ 1736, $1683 \mathrm{~cm}^{-1}(\mathrm{C}=\mathrm{O}) ;{ }^{1} \mathrm{H}-\mathrm{NMR}\left(600 \mathrm{MHz}, \mathrm{CDCl}_{3}\right): \delta 6.98(\mathrm{~d}, J=8.7 \mathrm{~Hz}, 2 \mathrm{H}, \mathrm{H}-\mathrm{Ar}), 6.81$ $(\mathrm{d}, J=8.7 \mathrm{~Hz}, 2 \mathrm{H}, \mathrm{H}-\mathrm{Ar}), 6.48$ (s, 1H, H-10), $6.14(\mathrm{~s}, 1 \mathrm{H}, \mathrm{H}-7), 5.88$ (s, 1H, H-6), 4.65-4.62 (m, 1H, 2- $\left.\mathrm{CH}_{2}\right)$, $3.80\left(\mathrm{~s}, 3 \mathrm{H}, \mathrm{OCH}_{3}\right), 3.79\left(\mathrm{~s}, 3 \mathrm{H}, \mathrm{OCH}_{3}\right), 3.72\left(\mathrm{~s}, 3 \mathrm{H}, \mathrm{OCH}_{3}\right), 3.61\left(\mathrm{~s}, 3 \mathrm{H}, \mathrm{OCH}_{3}\right), 3.60\left(\mathrm{~s}, 3 \mathrm{H}, \mathrm{OCH}_{3}\right), 3.35$ $\left(\mathrm{ddd}, J=15.1,8.7,1.8 \mathrm{~Hz}, 1 \mathrm{H}, 1-\mathrm{CH}_{2}\right), 2.86\left(\mathrm{ddd}, J=8.7,11.4,16.2 \mathrm{~Hz}, 1 \mathrm{H}, 2-\mathrm{CH}_{2}\right), 2.56-2.53(\mathrm{~m}, 1 \mathrm{H}$, 1- $\left.\mathrm{CH}_{2}\right), 2.52$ (s, 3H, N-CH3); ${ }^{13} \mathrm{C}-\mathrm{NMR}\left(150 \mathrm{MHz}, \mathrm{CDCl}_{3}\right): \delta 168.2,166.2,160.8,157.8,156.7,147.9$, 147.4, 129.6, 127.4 (2C), 126.4, 126.2, 115.8, 114.2 (2C), 113.8, 99.8, 55.9, 55.6, 55.5, 55.2, 52.5, 51.8, 43.2, 38.3, 32.8; LCMS $m / z$ : $524[\mathrm{M}+\mathrm{H}]^{+}$. Elemental analysis: calcd. for $\mathrm{C}_{26} \mathrm{H}_{29} \mathrm{~N}_{5} \mathrm{O}_{7} \mathrm{C} 59.65, \mathrm{H} 5.58, \mathrm{~N}$ $13.38 \%$, found C $59.83, \mathrm{H} 5.71, \mathrm{~N} 13.45 \%$.

Methyl (8E)-10-[1-(4-methoxyphenyl)-1H-tetrazol-5-yl]-7,11-dimethyl-5,6,7,10-tetrahydro[1,3]dioxolo[4,5-i] [3]benzazocine-9-carboxylate (2d), Yield $0.93 \mathrm{~g}(97 \%)$; white solid; m.p. $=167-169{ }^{\circ} \mathrm{C} ; R_{\mathrm{f}}=0.35$ (EtOAc-hexane, 2:1); IR (KBr) v $1681 \mathrm{~cm}^{-1}(\mathrm{C}=\mathrm{O}) ;{ }^{1} \mathrm{H}-\mathrm{NMR}\left(600 \mathrm{MHz}, \mathrm{CDCl}_{3}\right): \delta 7.42(\mathrm{~m}, 1 \mathrm{H}, \mathrm{H}-8)$, $7.16(\mathrm{~d}, J=8.8 \mathrm{~Hz}, 2 \mathrm{H}, \mathrm{H}-\mathrm{Ar}), 6.90(\mathrm{~d}, J=8.8 \mathrm{~Hz}, 2 \mathrm{H}, \mathrm{H}-\mathrm{Ar}), 6.85$ (s, 1H, H-10), 6.30 (s, 1H, H-4), 5.88 $\left(\mathrm{d}, J=1.5 \mathrm{~Hz}, 1 \mathrm{H}, 2-\mathrm{CH}_{2}\right), 5.83\left(\mathrm{~d}, J=1.5 \mathrm{~Hz}, 1 \mathrm{H}, 2-\mathrm{CH}_{2}\right), 4.03\left(\mathrm{ddd}, J=15.3,9.8,4.8 \mathrm{~Hz}, 1 \mathrm{H}, 6-\mathrm{CH}_{2}\right)$, $3.84\left(\mathrm{~s}, 3 \mathrm{H}, \mathrm{OCH}_{3}\right), 3.83\left(\mathrm{~s}, 3 \mathrm{H}, \mathrm{OCH}_{3}\right), 3.55\left(\mathrm{~s}, 3 \mathrm{H}, \mathrm{OCH}_{3}\right), 3.08\left(\mathrm{dt}, J=15.3,5.3 \mathrm{~Hz}, 1 \mathrm{H}, 6-\mathrm{CH}_{2}\right), 2.92$ (s, 3H, N-CH3), 2.90-2.89 (m, 1H, 5-CH $), 2.80\left(\mathrm{dt}, J=16.3,5.3 \mathrm{~Hz}, 1 \mathrm{H}, 5-\mathrm{CH}_{2}\right) ;{ }^{13} \mathrm{C}-\mathrm{NMR}(150 \mathrm{MHz}$, $\mathrm{CDCl}_{3}$ ): $\delta 167.0,160.6,160.0,153.5,147.8,141.2,135.6,132.3,127.1$ (2C), 126.9, 121.6, 113.9 (2C), 105.3, 101.0, 99.3, 59.9, 55.6, 52.4, 51.1, 45.0, 34.9, 30.0; LCMS m/z: $480[\mathrm{M}+\mathrm{H}]^{+}$. Elemental analysis: calcd. for $\mathrm{C}_{24} \mathrm{H}_{25} \mathrm{~N}_{5} \mathrm{O}_{6} \mathrm{C} 60.12$, H 5.26, N 14.61\%, found C 59.89, H 5.46, N $14.52 \%$.

1-\{(8E)-11-Methoxy-10-[1-(4-methoxyphenyl)-1H-tetrazol-5-yl]-7-methyl-5,6,7,10-tetrahydro[1,3]dioxolo[4,5-i] [3]benzazocin-9-yl\}ethanone (2e), Yield $0.88 \mathrm{~g}(95 \%)$; white solid; m.p. $=225-227{ }^{\circ} \mathrm{C} ; R_{\mathrm{f}}=0.42$ (EtOAc-hexane, 1:1); IR (KBr) v $1620 \mathrm{~cm}^{-1}(\mathrm{C}=\mathrm{O}) ;{ }^{1} \mathrm{H}-\mathrm{NMR}\left(600 \mathrm{MHz}, \mathrm{CDCl}_{3}\right): \delta 7.27$ (s, 1H, H-8); 7.18 (br.s, 1H, H-10), 7.15-7.12 (m, 2H, H-Ar), 6.87-6.85 (m, 2H, H-Ar), 6.28 (s, 1H, H-4), 5.87 (d, J = 1.4 Hz, $\left.1 \mathrm{H}, 2-\mathrm{CH}_{2}\right), 5.81\left(\mathrm{~d}, J=1.4 \mathrm{~Hz}, 1 \mathrm{H}, 2-\mathrm{CH}_{2}\right), 4.15-4.10\left(\mathrm{~m}, 1 \mathrm{H}, 6-\mathrm{CH}_{2}\right), 3.83\left(\mathrm{~s}, 3 \mathrm{H}, \mathrm{OCH}_{3}\right), 3.80(\mathrm{~s}, 3 \mathrm{H}$, $\left.\mathrm{OCH}_{3}\right), 3.10\left(\mathrm{ddd}, J=15.6,6.4,4.6 \mathrm{~Hz}, 1 \mathrm{H}, 6-\mathrm{CH}_{2}\right), 2.96(\mathrm{~s}, \mathrm{H}, \mathrm{N}-\mathrm{CH} 3), 2.87$ (ddd, $J=16.6,10.2,6.4$ $\left.\mathrm{Hz}, 1 \mathrm{H}, 5-\mathrm{CH}_{2}\right), 2.67\left(\mathrm{dt}, J=16.6,4.6 \mathrm{~Hz}, 1 \mathrm{H}, 5-\mathrm{CH}_{2}\right), 2.06\left(\mathrm{~s}, 3 \mathrm{H}, \mathrm{CH}_{3}\right) ;{ }^{13} \mathrm{C}-\mathrm{NMR}\left(150 \mathrm{MHz}, \mathrm{CDCl}_{3}\right)$ : $\delta 192.7 ; 160.3,159.7,155.3,147.6,141.1,135.4,131.6,126.7,126.7$ (2C), 121.4, 113.7 (2C), 107.7, 104.9, 
100.7, 59.6, 55.4, 52.1, 44.7, 34.5, 27.9, 24.5; LCMS $m / z: 464[\mathrm{M}+\mathrm{H}]^{+}$. Elemental analysis: calcd. for $\mathrm{C}_{24} \mathrm{H}_{25} \mathrm{~N}_{5} \mathrm{O}_{5} \mathrm{C} 62.19, \mathrm{H} 5.44, \mathrm{~N} 15.11 \%$, found C 61.93, H 5.63, N $15.26 \%$.

Dimethyl (8E)-11-methoxy-10-[1-(4-methoxyphenyl)-1H-tetrazol-5-yl]-7-methyl-5,6,7,10-tetrahydro[1,3]dioxolo [4,5-i][3]benzazocin-8,9-dicarboxylate (2f), Yield $1.03 \mathrm{~g}(96 \%)$; white solid; m.p. $=212-214{ }^{\circ} \mathrm{C} ; R_{\mathrm{f}}=0.47$ (EtOAc-hexane, 1:1); IR (KBr) v 1732, $1691 \mathrm{~cm}^{-1}(\mathrm{C}=\mathrm{O}) ;{ }^{1} \mathrm{H}-\mathrm{NMR}\left(600 \mathrm{MHz}, \mathrm{CDCl}_{3}\right): \delta 7.13-7.12$ (m, 2H, H-Ar), 6.86-6.84 (m, 2H, H-Ar), 6.72 (s, 1H, H-10), 6.22 (s, 1H, H-4), 5.86 (d, J = 1.4 Hz, 1H, $\left.2-\mathrm{CH}_{2}\right), 5.78\left(\mathrm{~d}, J=1.4 \mathrm{~Hz}, 1 \mathrm{H}, 2-\mathrm{CH}_{2}\right), 4.35\left(\mathrm{ddd}, J=15.2,10.0,6.0 \mathrm{~Hz}, 1 \mathrm{H}, 6-\mathrm{CH}_{2}\right), 3.81\left(\mathrm{~s}, 3 \mathrm{H}, \mathrm{OCH}_{3}\right)$, $3.75\left(\mathrm{~s}, 3 \mathrm{H}, \mathrm{OCH}_{3}\right), 3.72\left(\mathrm{~s}, 3 \mathrm{H}, \mathrm{OCH}_{3}\right), 3.58\left(\mathrm{~s}, 3 \mathrm{H}, \mathrm{OCH}_{3}\right), 3.30\left(\mathrm{ddd}, J=15.2,6.7,4.0 \mathrm{~Hz}, 1 \mathrm{H}, 6-\mathrm{CH}_{2}\right)$, $2.87\left(\mathrm{ddd}, J=16.7,10.0,6.7 \mathrm{~Hz}, 1 \mathrm{H}, 5-\mathrm{CH}_{2}\right), 2.53(\mathrm{~s}, 3 \mathrm{H}, \mathrm{N}-\mathrm{CH} 3), 2.45-2.41\left(\mathrm{~m}, 1 \mathrm{H}, 5-\mathrm{CH}_{2}\right) ;{ }^{13} \mathrm{C}-\mathrm{NMR}$ $\left(150 \mathrm{MHz}, \mathrm{CDCl}_{3}\right): \delta 167.5 ; 166.4,160.6,158.3,155.6,148.1,141.1,135.7,132.6,126.8(2 \mathrm{C}), 126.5,120.4$, 114.0 (2C), 105.1, 101.1, 99.7, 60.1, 55.9, 55.6, 52.4, 51.7, 38.8, 32.62, 32.60; LCMS $m / z: 538[\mathrm{M}+\mathrm{H}]^{+}$. Elemental analysis: calcd. for $\mathrm{C}_{26} \mathrm{H}_{27} \mathrm{~N}_{5} \mathrm{O}_{8} \mathrm{C} 58.10, \mathrm{H} 5.06, \mathrm{~N} 13.03 \%$, found C 57.83, H 5.21, N $12.90 \%$.

\subsection{The Interaction of Isoquinoline $\mathbf{1} \mathbf{c}$ with $D M A D$}

$\operatorname{DMAD}(0.44 \mathrm{~g}, 3.1 \mathrm{mmol})$ was added to a solution of isoquinoline $1 \mathrm{c}(0.5 \mathrm{~g}, 1.56 \mathrm{mmol})$ in trifluoroethanol $(5 \mathrm{~mL})$. The mixture was kept at $20{ }^{\circ} \mathrm{C}$ for 2 days. The reaction progress was monitored by TLC (sorbfil, EtOAc-hexane, 1:3). The solvent was evaporated in vacuum, and the residue was chromatographed on a silica gel column. Azocine $\mathbf{2} \mathbf{g}$ and spiro compound $\mathbf{3}$ were eluted with EtOAc-hexane, 1:2, and recrystallized from EtOAc-hexane mixture.

Dimethyl 6-[1-(4-methoxyphenyl)-1H-tetrazol-5-yl]-3-methyl-1,2,3,6-tetrahydro-3-benzazocin-4,5-dicarboxylate (2g), Yield $0.36 \mathrm{~g}(50 \%)$; white solid; m.p. $=125-127^{\circ} \mathrm{C} ; R_{\mathrm{f}}=0.70$ (EtOAc-hexane, 1:2); $\mathrm{IR}(\mathrm{KBr}) \vee 1734$, $1681 \mathrm{~cm}^{-1}(\mathrm{C}=\mathrm{O}) ;{ }^{1} \mathrm{H}-\mathrm{NMR}\left(600 \mathrm{MHz}, \mathrm{CDCl}_{3}\right): \delta 7.10-7.08$ (m, 1H, H-Ar); 7.02-6.97 (m, 4H, H-Ar), 6.81-6.80 (m, 3H, H-Ar), 6.03 (s, 1H, H-6), 4.60-4.53 (m, 1H, 2- $\left.\mathrm{CH}_{2}\right), 3.82\left(\mathrm{~s}, 3 \mathrm{H}, \mathrm{OCH}_{3}\right), 3.73(\mathrm{~s}, 3 \mathrm{H}$, $\left.\mathrm{OCH}_{3}\right), 3.63\left(\mathrm{~s}, 3 \mathrm{H}, \mathrm{OCH}_{3}\right), 3.39\left(\mathrm{dd}, J=13.8,8.1 \mathrm{~Hz}, 1 \mathrm{H}, 2-\mathrm{CH}_{2},\right), 2.99-2.93\left(\mathrm{~m}, 1 \mathrm{H}, 1-\mathrm{CH}_{2}\right), 2.62(\mathrm{dd}$, $\left.J=15.9,6.8 \mathrm{~Hz}, 1 \mathrm{H}, 1-\mathrm{CH}_{2},\right), 2.49$ (s, 3H, N-CH3); ${ }^{13} \mathrm{C}-\mathrm{NMR}\left(150 \mathrm{MHz}, \mathrm{DMSO}-d_{6}\right): \delta 167.4,165.6,160.3$, 157.6, 155.8, 137.2, 134.2, 132.3, 130.9, 127.5, 127.1 (2C), 127.0, 126.0, 114.3 (2C), 99.4, 55.6, 54.0, 52.4, 51.6, 42.6, 38.2, 32.6; LCMS m/z: 464 [M + H] $]^{+}$. Elemental analysis: calcd. for $\mathrm{C}_{24} \mathrm{H}_{25} \mathrm{~N}_{5} \mathrm{O}_{5} \mathrm{C}$ 62.19, $\mathrm{H}$ 5.44, N $15.11 \%$, found C 61.93, H $5.69, \mathrm{~N} 15.28 \%$.

Dimethyl (11E)-11-\{[1-(4-methoxyphenyl)-1H-tetrazol-5-yl]methylidene\}-3-methyl-3-azaspiro[5.5]undeca-1,7,9triene-1,2-dicarboxylate (3), Yield $0.12 \mathrm{~g}(17 \%)$; white solid; m.p. $=126-127^{\circ} \mathrm{C} ; R_{\mathrm{f}}=0.50$ (EtOAc-hexane, 1:1); IR $(\mathrm{KBr}) \vee 1736,1680 \mathrm{~cm}^{-1}(\mathrm{C}=\mathrm{O}) ;{ }^{1} \mathrm{H}-\mathrm{NMR}\left(600 \mathrm{MHz}, \mathrm{CDCl}_{3}\right): \delta 7.73(\mathrm{~d}, J=9.7 \mathrm{~Hz}, 1 \mathrm{H}$, H-cyclohexadiene); $7.34(\mathrm{~d}, J=8.7 \mathrm{~Hz}, 2 \mathrm{H}, \mathrm{H}-\mathrm{Ar}), 7.04(\mathrm{~d}, J=8.7 \mathrm{~Hz}, 2 \mathrm{H}, \mathrm{H}-\mathrm{Ar}), 6.31(\mathrm{dd}, J=9.7,5.5 \mathrm{~Hz}$, $1 \mathrm{H}, \mathrm{H}$-cyclohexadiene), 6.03-5.99 (m, 2H, H-cyclohexadiene), 5.91 (s, $1 \mathrm{H},=\mathrm{CH}-\mathrm{Ar}), 3.88\left(\mathrm{~s}, 3 \mathrm{H}, \mathrm{OCH}_{3}\right)$, $3.83\left(\mathrm{~s}, 3 \mathrm{H}, \mathrm{OCH}_{3}\right), 3.54\left(\mathrm{~s}, 3 \mathrm{H}, \mathrm{OCH}_{3}\right), 3.22\left(\mathrm{ddd}, J=13.1,9.6,3.9 \mathrm{~Hz}, 1 \mathrm{H}, 4-\mathrm{CH}_{2}\right), 2.97(\mathrm{ddd}, J=13.1$, $\left.4.8,4.6 \mathrm{~Hz}, 1 \mathrm{H}, 4-\mathrm{CH}_{2}\right), 2.75(\mathrm{~s}, 3 \mathrm{H}, \mathrm{N}-\mathrm{CH} 3), 1.87\left(\mathrm{ddd}, J=13.6,9.6,3.9 \mathrm{~Hz}, 1 \mathrm{H}, 5-\mathrm{CH}_{2},\right), 1.81-1.77$ (m, $\left.1 \mathrm{H}, 5-\mathrm{CH}_{2}\right) ;{ }^{13} \mathrm{C}-\mathrm{NMR}\left(150 \mathrm{MHz}, \mathrm{CDCl}_{3}\right): \delta 166.6,165.6,160.5,156.4,151.2,150.7,128.5,142.0$, 126.7, 126.2 (2C), 122.0, 119.4, 114.8 (2C), 105.3, 97.4, 55.6, 52.6, 50.9, 42.5 (2C), 39.5, 33.7, LCMS $m / z$ : $464[\mathrm{M}+\mathrm{H}]^{+}$. Elemental analysis: calcd. for $\mathrm{C}_{24} \mathrm{H}_{25} \mathrm{~N}_{5} \mathrm{O}_{5} \mathrm{C} 62.19, \mathrm{H} 5.44, \mathrm{~N} 15.11 \%$, found $\mathrm{C} 61.43$, H 5.72, N 15.21\%.

\subsection{X-ray Structure Determination of Compound 3}

The structures of product 3 were unambiguously established by X-ray diffraction study and are shown in Figure 1 along with the atomic numbering schemes. The tetrahydropyridine ring of the spiro compound 3 assumes a slightly distorted "sofa" conformation with the carbon atom $C(16)$ extending from the plane formed by the remaining atoms of the ring by $0.658 \AA$. Carbonyl fragment $\mathrm{C}(19)-\mathrm{O}(3)$ of the ester group is practically coplanar with the basal plane of the tetrahydropyridine ring $C(15)-C(18)=C(21)-N(5)-C(17)$ (the dihedral angle is equal to $\left.6.40^{\circ}\right)$ due to the conjugation of bonds. The nitrogen atom N(5) has a trigonal planar configuration (the sum of the valence angles is equal to $359.7^{\circ}$ ). The cyclohexadiene ring of the spiro compound 3 assumes a slightly distorted "sofa" 
conformation with the carbon atom $\mathrm{C}(15)$ extending from the plane formed by the remaining atoms of the ring by $0.444 \AA$. The 4-methoxyphenyl substituent in molecule 3 is twisted with a tetrazole ring at an angle of $34.20^{\circ}$. The molecule of spiro compound 3 contains the asymmetric carbon atom $\mathrm{C}(15)$. The molecules of compound 3 in the crystal form centrosymmetric dimers due to two intermolecular hydrogen bonds $\mathrm{C}(5)-\mathrm{H}(5) \cdots \mathrm{O}(5)^{*}$.

The crystals of compound $3\left(\mathrm{C}_{24} \mathrm{H}_{25} \mathrm{~N}_{5} \mathrm{O}_{5}, M 463.49\right)$ are triclinic, space group $P-1$ at $120 \mathrm{~K}: a=8.2773(9), b=11.6273(13), c=11.9759(12) \AA ; \beta=94.980(2)^{\circ} ; V 1097.7(2) \AA^{3} ; Z 2$; $d_{\text {calc }} 1.402 \mathrm{~g} \mathrm{~cm}^{-3} ; F(000) 488.0 ; \mu 0.101 \mathrm{~mm}^{-1}$. The unit cell parameters and intensity of 8547 reflections (4994 independent reflections, $R_{\text {int }}$ 0.0235) were measured on an automatic three-circle diffractometer Bruker SMART APEX-II CCD (MoKa radiation $(\lambda=0.71073 \AA$ ) , graphite-monochromator, $\omega$-scanning, $\left.3.57^{\circ} \leq 2 \Theta \leq 54.968^{\circ}\right)$.

The structure was determined by the direct method and refined by the least-squares technique in the full-matrix anisotropic approximation for non-hydrogen atoms based on $F^{2}$. The positions of hydrogen atoms were calculated geometrically and were included in the refinement with fixed positional parameters (the "rider" model) and with isotropic displacement parameters $\left(U_{\text {iso }}(\mathrm{H})=1.5 \mathrm{U}_{\text {eq }}(\mathrm{C})\right.$ for $\mathrm{CH}_{3}$ groups and $1.2 U_{\mathrm{eq}}(\mathrm{C})$ for other groups $)$. The final probability factors were $R_{1}=0.044(I \geq 2 \sigma(I))$ and $w R_{2}=0.106$ for all independent reflections. All calculations were carried out using the programs OLEX-2 [20] and SHELXTL [21] software package. Tables of the coordinates of atoms, bond lengths, valence and torsion angles, and anisotropic temperature parameters of compound 3 were deposited at the Cambridge Crystallographic Data Center (deposit CCDC 1848342).

\subsection{The Interaction of Isoquinoline 1c with Methyl Propiolate}

Methyl propiolate $(0.52 \mathrm{~g}, 6.2 \mathrm{mmol})$ was added to a solution of isoquinoline $1 \mathrm{c}(0.5 \mathrm{~g}, 1.56 \mathrm{mmol})$ in trifluoroethanol $(7 \mathrm{~mL})$. The reaction mixture was heated under reflux for $120 \mathrm{~h}$. The reaction progress was monitored by TLC (sorbfil, EtOAc-hexane, 1:3). The solvent was evaporated in vacuum. The residue was chromatographed on a silica gel column. Vinylisoquinoline $\mathbf{4 a}$ and benzazocine $\mathbf{2 h}$ were eluted with EtOAc-hexane, 1:2, and recrystallized from EtOAc-hexane mixture.

Methyl (4E)-6-[1-(4-methoxyphenyl)-1H-tetrazol-5-yl]-3-methyl-1,2,3,6-tetrahydro-3-benzazocin-5-carboxylate (2h), Yiled $0.14 \mathrm{~g}(22 \%) ;$ white solid; m.p. = $139-140{ }^{\circ} \mathrm{C} ; R_{\mathrm{f}}=0.37$ (EtOAc-hexane, 1:1); IR $(\mathrm{KBr}) v$ $1679 \mathrm{~cm}^{-1}(\mathrm{C}=\mathrm{O}){ }^{1} \mathrm{H}-\mathrm{NMR}\left(600 \mathrm{MHz}, \mathrm{CDCl}_{3}\right): \delta 7.37$ (s, 1H, H-4), 7.13-7.10 (m, 4H, H-Ar), 7.05-7.02 (m, 1H, H-Ar), 6.96 (d, J = 7.8 Hz, 1H, H-Ar), 6.86 (d, J = 8.7 Hz, 2H, H-Ar), 6.13 (s, 1H, H-6), 3.88-3.84 $\left(\mathrm{m}, 1 \mathrm{H}, 2-\mathrm{CH}_{2}\right), 3.83\left(\mathrm{~s}, 3 \mathrm{H}, \mathrm{OCH}_{3}\right), 3.53\left(\mathrm{~s}, 3 \mathrm{H}, \mathrm{OCH}_{3}\right), 3.13-3.03\left(\mathrm{~m}, 3 \mathrm{H}, 1,2-\mathrm{CH}_{2}\right), 2.91(\mathrm{~s}, 3 \mathrm{H}, \mathrm{N}-\mathrm{CH} 3)$; ${ }^{13} \mathrm{C}-\mathrm{NMR}\left(150 \mathrm{MHz}_{,} \mathrm{CDCl}_{3}\right): \delta 169.3,160.8,159.4,153.6,136.6,136.1,132.4,131.8,127.7$ (2C), 127.32 , 127.26, 126.7, 114.1 (2C), 94.3, 55.6, 51.32, 51.25, 44.2, 41.3, 35.7; LCMS m/z: 406 [M + H] $]^{+}$Elemental analysis: calcd. for $\mathrm{C}_{22} \mathrm{H}_{23} \mathrm{~N}_{5} \mathrm{O}_{3} \mathrm{C}$ 65.17, $\mathrm{H} 5.72, \mathrm{~N} \mathrm{17.27 \%}$, found C 65.36, H 5.95, N 17.03\%.

Methyl (2E)-3-\{1-[1-(4-methoxyphenyl)-1H-tetrazol-5-yl]-2-methyl-1,2,3,4-tetrahydroisoquinolin-1-yl\}prop-2enoate (4a), Yield $0.063 \mathrm{~g}(10 \%)$; white solid; m.p. $=132-134{ }^{\circ} \mathrm{C} ; R_{\mathrm{f}}=0.54$ (EtOAc-hexane, 1:2); IR $(\mathrm{KBr}) v$ $1681 \mathrm{~cm}^{-1}(\mathrm{C}=\mathrm{O}) ;{ }^{1} \mathrm{H}-\mathrm{NMR}\left(600 \mathrm{MHz}, \mathrm{CDCl}_{3}\right): \delta 7.84\left(\mathrm{~d}, \mathrm{~J}=16.0 \mathrm{~Hz}, 1 \mathrm{H}, \mathrm{CH}=\mathrm{CHCO}_{2} \mathrm{CH}_{3}\right), 7.11-7.09$ (m, 1H, H-Ar), 7.01 (t, J = 7.5 Hz, 1H, H-Ar), 6.90 (d, J = 7.5 Hz, 1H, H-Ar), $6.66(\mathrm{~d}, J=8.7 \mathrm{~Hz}, 2 \mathrm{H}$, H-Ar), 6.58-6.55 (m, 3H, H-Ar), $5.36\left(\mathrm{~d}, \mathrm{~J}=16.0 \mathrm{~Hz}, 1 \mathrm{H}, \mathrm{C} \underline{\mathrm{H}}=\mathrm{CHCO}_{2} \mathrm{CH}_{3}\right), 3.77\left(\mathrm{~s}, 3 \mathrm{H}, \mathrm{OCH}_{3}\right), 3.73$ $\left(\mathrm{s}, 3 \mathrm{H}, \mathrm{OCH}_{3}\right), 2.64-2.59\left(\mathrm{~m}, 1 \mathrm{H}, 3-\mathrm{CH}_{2}\right), 2.52\left(\mathrm{dd}, J=12.6,5.1 \mathrm{~Hz}, 1 \mathrm{H}, 4-\mathrm{CH}_{2}\right), 2.42-2.40\left(\mathrm{~m}, 1 \mathrm{H}, 3-\mathrm{CH}_{2}\right)$, 2.24-2.20 (m, 1H, 4-CH2), 2.18 (s, 3H, N-CH3); ${ }^{13} \mathrm{C}-\mathrm{NMR}\left(150 \mathrm{MHz}, \mathrm{CDCl}_{3}\right): \delta 165.8,160.3,158.5,144.5$, 135.5, 133.3, 128.7 (2C), 127.8, 127.51 (2C), 127.46, 126.6, 126.2, 113.2 (2C), 63.4, 55.5, 51.7, 45.5, 38.8, 28.5; LCMS m/z: $406[\mathrm{M}+\mathrm{H}]^{+}$. Elemental analysis: calcd. for $\mathrm{C}_{22} \mathrm{H}_{23} \mathrm{~N}_{5} \mathrm{O}_{3} \mathrm{C}$ 65.17, $\mathrm{H}$ 5.72, $\mathrm{N} 17.27 \%$, found C 64.42, H 5.89, N 17.41\%.

\subsection{The Interaction of Isoquinoline 1c with Acetylacetylene}

Acetylacetylene $(0.54 \mathrm{~g}, 7.9 \mathrm{mmol})$ was added to a solution of isoquinoline $1 \mathrm{c}(0.25 \mathrm{~g}, 0.78 \mathrm{mmol})$ in trifluoroethanol $(5 \mathrm{~mL})$. The reaction mixture was refluxed for 180 hours. The reaction progress 
was monitored by TLC (sorbfil, EtOAc-hexane, 1:3). The solvent was evaporated in vacuum. The residue was purified by column chromatography on silica gel. Isoquinoline $4 \mathbf{b}$ was eluted with EtOAc-hexane, 1:3.

(3E)-4-\{1-[1-(4-Methoxyphenyl)-1H-tetrazol-5-yl]-2-methyl-1,2,3,4-tetrahydroisoquinolin-1-yl\}but-3-ene-2-one (4b), Yiled $0.052 \mathrm{~g}(17 \%) ;$ white solid; m.p. $=132-134{ }^{\circ} \mathrm{C} ; R_{\mathrm{f}}=0.40$ (EtOAc-hexane, $\left.1: 2\right) ; \mathrm{IR}(\mathrm{KBr})$ $v 1630 \mathrm{~cm}^{-1}(\mathrm{C}=\mathrm{O}) ;{ }^{1} \mathrm{H}-\mathrm{NMR}\left(600 \mathrm{MHz}, \mathrm{CDCl}_{3}\right): \delta 7.73(\mathrm{~d}, J=16.5 \mathrm{~Hz}, 1 \mathrm{H}, \mathrm{C} \underline{\mathrm{H}}=\mathrm{CH}-\mathrm{Ac}), 7.13$ $(\mathrm{t}, J=7.5 \mathrm{~Hz}, 1 \mathrm{H}, \mathrm{H}-\mathrm{Ar}), 7.03(\mathrm{t}, J=7.9 \mathrm{~Hz}, 1 \mathrm{H}, \mathrm{H}-\mathrm{Ar}), 6.92(\mathrm{~d}, J=7.5 \mathrm{~Hz}, 1 \mathrm{H}, \mathrm{H}-\mathrm{Ar}), 6.69$ (d, $J=9.1$ $\mathrm{Hz}, 2 \mathrm{H}, \mathrm{H}-\mathrm{Ar}), 6.63$ (d, J = 9.1 Hz, 2H, H-Ar), 6.59 (d, J = 7.9 Hz, 1H, H-Ar), 5.58 (d, J = 16.5 Hz, 1H, $\mathrm{CH}=\mathrm{CH}-\mathrm{Ac}), 3.80\left(\mathrm{~s}, 3 \mathrm{H}, \mathrm{OCH}_{3}\right), 2.73-2.64\left(\mathrm{~m}, 1 \mathrm{H}, 3-\mathrm{CH}_{2}\right), 2.60-2.54\left(\mathrm{~m}, 1 \mathrm{H}, 4-\mathrm{CH}_{2}\right), 2.49-2.46(\mathrm{~m}, 1 \mathrm{H}$, 3- $\left.\mathrm{CH}_{2}\right), 2.39(\mathrm{~m}, 3 \mathrm{H}, \mathrm{N}-\mathrm{CH} 3), 2.31-2.20\left(\mathrm{~m}, 4 \mathrm{H}, \mathrm{COCH}_{3}\right.$ and $\left.4-\mathrm{CH}_{2}\right) ;{ }^{13} \mathrm{C}-\mathrm{NMR}\left(150 \mathrm{MHz}, \mathrm{CDCl}_{3}\right)$ : $\delta$ 198.3, 160.5, 158.2, 143.7, 136.2, 135.5, 133.2, 128.9, 128.8, 128.1, 127.7 (2C), 127.5, 126.5, 113.4 (2C), 63.7, 55.7, 45.8, 39.0, 28.5, 27.1; LCMS m/z: $390[\mathrm{M}+\mathrm{H}]^{+}$. Elemental analysis: calcd. for $\mathrm{C}_{22} \mathrm{H}_{23} \mathrm{~N}_{5} \mathrm{O}_{2}$ C 67.85, H 5.95, N 17.98\%, found C 67.98, H 6.21, N 18.10\%.

Supplementary Materials: The following are available online. ${ }^{1} \mathrm{H}$ and ${ }^{13} \mathrm{C}-\mathrm{NMR}$ spectra of all the products $1-4$ Figures S1-S29.

Author Contributions: L.G.V., E.V.V.d.E., and A.A.T. conceived and designed the experiments; R.S., E.V.A., T.A.D.T., V.T.N., and T.A.L. performed the experiments; T.N.B. analyzed the data; and A.V.V. wrote the paper.

Funding: The publication was prepared with the support of the "RUDN University Program 5-100" and by the Russian Foundation for Basic Research (grant 17-53-540001) and Vietnam (VAST.HTQT.NGA.04/17-18).

Conflicts of Interest: The authors declare no conflict of interest.

\section{References}

1. Soltanzadeh, Z.; Imanzadeh, G.; Noroozi-Pesyan, N.; Şahin, E. Green synthesis of pyrazole systems under solvent-free conditions. Green Chem. Lett. Rev. 2017, 10, 148-153. [CrossRef]

2. Bode, M.L.; Gravestock, D.; Rousseau, A.L. Synthesis, Reactions and Uses of Isocyanides in Organic Synthesis. An Update. Org. Prep. Proced. Int. 2016, 28, 89-221. [CrossRef]

3. Akbarzadeh, A.; Dekamin, M.G. A facile and environmentally benign polyethylene glycol 600-mediated method for the synthesis of densely functionalized 2-aminothiophene derivatives under ultrasonication. Green Chem. Lett. Rev. 2017, 10, 315-323. [CrossRef]

4. Kumar, A.; Saxena, D.; Gupta, M.K. Nanoparticle catalyzed reaction (NPCR): ZnO-NP catalyzed Ugi-reaction in aqueous medium. Green Chem. 2013, 15, 2699-2703. [CrossRef]

5. Edjlali, L.; Vessally, E.; Jafari, Z.; Esrafili, M.D. A novel multicomponent reaction between amino acids, aromatic aldehydes and ptoluenesulfonylmethyl isocyanide: An efficient and green one-pot synthesis using nanosilica. Green Chem. Lett. Rev. 2016, 9, 13-19. [CrossRef]

6. Aminimanesh, A.; Shirian, S. Synthesis of 5-Substituted Tetrazoles from Nitriles Catalyzed by Sulfamic Acid. Org. Prep. Proced. Int. 2017, 49, 346-354. [CrossRef]

7. Haldar, S.; Saha, S.; Mandal, S.; Jana, C.K. C-H Functionalization Enabled Stereoselective Ugi-azide Reaction to $\alpha$-tetrazolyl Alicyclic Amines. Green Chem. 2018. [CrossRef]

8. Naeimi, H.; Kiani, F.; Moradian, M. Rapid microwave promoted heterocyclization of primary amines with triethyl orthoformate and sodium azide using zinc sulfide nanoparticles as recyclable catalyst. Green Chem. Lett. Rev. 2018, 11, 361-369. [CrossRef]

9. Pathare, R.S.; Ansari, A.J.; Verma, S.; Maurya, A.; Maurya, A.K.; Agnihotri, V.K.; Sharon, A.; Pardasani, R.T.; Sawant, D.M. Sequential Pd(0)/Fe(III) catalyzed azide-Isocyanide Coupling/Cyclization Reaction: One-pot synthesis of Aminotetrazoles. J. Org. Chem. 2018. [CrossRef] [PubMed]

10. Verma, F.; Sahu, A.; Singh, P.K.; Rai, A.; Singh, M.; Rai, V.K. Visible-light driven regioselective synthesis of 1H-tetrazoles from aldehydes through isocyanide-based[3 + 2]cycloaddition. Green Chem. 2018. [CrossRef]

11. Mofakham, H.; Shaabani, A.; Mousavifaraz, S.; Hajishaabanha, F.; Shaabani, S.; Weng Ng, S. A novel one-pot pseudo-five-component condensation reaction towards bifunctional diazepine-tetrazole containing compounds: Synthesis of $1 H$-tetrazolyl-1H-1,4-diazepine-2,3-dicarbonitriles and $1 H$-tetrazolyl-benzo[b][1,4]diazepines. Mol. Divers. 2012, 16, 351-356. [CrossRef] [PubMed] 
12. Rajasekaran, A.; Thampi, P.P. Synthesis and analgesic evaluation of some 5-[ß-(10-phenothiazinyl)ethyl]-1-(acyl)-1,2,3,4-tetrazoles. Eur. J. Med. Chem. 2004, 39, 273-279. [CrossRef] [PubMed]

13. Bhaskar, V.H.; Mohite, P.B. Synthesis, characterization and evaluation of anticancer activity of some tetrazole derivatives. J. Optoelectron. Biomed. Mater. 2010, 2, 249-259.

14. Carotti, A.; Candia, M.; Catto, M.; Borisova, T.N.; Varlamov, A.V.; Méndez-Álvarez, E.; Soto-Otero, R.; Voskressensky, L.G.; Altomare, C. Ester derivatives of annulated tetrahydroazocines: A new class of selective acetylcholinesterase inhibitors. Bioorg. Med. Chem. 2006, 14, 7205-7212. [CrossRef] [PubMed]

15. Voskressensky, L.G.; Borisova, T.N.; Kulikova, L.N.; Varlamov, A.V.; Catto, M.; Altomare, C.; Carotti, A. Tandem cleavage of hydrogenated $\beta$ - and $\gamma$-carbolines-new practical synthesis of tetrahydroazocino[ $[4,5-b]$ indoles and tetrahydroazocino $[5,4-b]$ indoles showing acetylcholinesterase inhibitory activity. Eur. J. Org. Chem. 2004, 14, 3128-3135. [CrossRef]

16. Borisov, R.S.; Voskressensky, L.G.; Polyakov, A.I.; Borisova, T.N.; Varlamov, A.V. A concise approach toward tetrazolyl-substituted benzazocines via a novel isocyanide-based multicomponent reaction. Synlett 2014, 25, 955-958. [CrossRef]

17. Voskressensky, L.G.; Titov, A.A.; Samavati, R.; Kobzev, M.S.; Dorovatovskii, P.V.; Khrustalev, V.N.; Hong, H.C.; An Dang Thi, T.; Nguyen Van, T.; Sorokina, E.A.; et al. Synthesis of 1-tetrazolyl-substituted 2,3,4,9-tetrahydro- $1 H$ - $\beta$-carbolines and their transformations involving activated alkynes. Chem. Heterocycl. Compd. 2017, 53, 575-581. [CrossRef]

18. Voskressensky, L.G.; Kovaleva, S.A.; Borisova, T.N.; Listratova, A.V.; Eresko, A.B.; Tolkunov, V.S.; Tolkunov, S.V.; Varlamov, A.V. Tandem transformations of tetrahydrobenzothieno[2,3-c]piridines in the presence of activated alkynes. Tetrahedron 2010, 66, 9421-9430. [CrossRef]

19. Voskressensky, L.G.; Borisova, T.N.; Kovaleva, S.A.; Listratova, A.V.; Kulikova, L.N.; Khrustalev, V.N.; Ovcharov, M.V.; Varlamov, A.V. Transformations of tetrahydropyrido $\left[4^{\prime}, 3^{\prime}: 4,5\right]$ thieno[2,3- $\left.d\right]$ pyrimidin-4(3H)-ones in the presence of alkynes bearing electron-withdrawing substituents. Russ. Chem. Bull. Int. Ed. 2012, 61, 370-379. [CrossRef]

20. Dolomanov, O.V.; Bourhis, L.J.; Gildea, R.J.; Howard, J.A.K.; Puschmann, H. OLEX2: A complete structure solution, refinement and analysis program. J. Appl. Cryst. 2009, 42, 339-341. [CrossRef]

21. Sheldrick, G.M. Crystal structure refinement with SHELXL. Acta Cryst. 2015, 71, 3-8. [CrossRef]

Sample Availability: Samples of the compounds are available from the authors.

(C) 2018 by the authors. Licensee MDPI, Basel, Switzerland. This article is an open access article distributed under the terms and conditions of the Creative Commons Attribution (CC BY) license (http:// creativecommons.org/licenses/by/4.0/). 